PROCEEDINGS OF THE

AMERICAN MATHEMATICAL SOCIETY

Volume 126, Number 6, June 1998, Pages 1579-1581

S $0002-9939(98) 04546-8$

\title{
A SIMPLE AND DIRECT DERIVATION FOR THE NUMBER OF NONCROSSING PARTITIONS
}

\author{
S. C. LIAW, H. G. YEH, F. K. HWANG, AND G. J. CHANG \\ (Communicated by Jeffry N. Kahn)
}

\begin{abstract}
Kreweras considered the problem of counting noncrossing partitions of the set $\{1,2, \cdots, n\}$, whose elements are arranged into a cycle in its natural order, into $p$ parts of given sizes $n_{1}, n_{2}, \cdots, n_{p}$ (but not specifying which part gets which size). He gave a beautiful and surprising result whose proof resorts to a recurrence relation. In this paper we give a direct, entirely bijective, proof starting from the same initial idea as Kreweras' proof.
\end{abstract}

\section{INTRODUCTION}

A noncrossing partition of the set $[n]=\{1,2, \cdots, n\}$, whose elements are arranged into a cycle in its natural order, is a partition $\pi$ of the set $[n]$ with the property that there do not exist four numbers $a<b<c<d$ such that $a$ and $c$ are in one part but $b$ and $d$ are in another part. The study of noncrossing partitions goes back at least to Becker [1], where they are called "planar rhyme schemes." The systematic study of noncrossing partitions began with Kreweras [7] and Poupard [10]. For some further work on noncrossing partitions, see [2], [3], [5], [6], [9], [10], [11], [12], [13], [14] and the references given there. Let $f\left(n_{1}, n_{2}, \cdots, n_{p}\right)$ denote the number of noncrossing partitions of $[n]$ into $p$ parts of given sizes $n_{1}, n_{2}, \cdots, n_{p}$ (but not specifying which part gets which size); and let $p_{k}$ denote the number of parts with size $k$. Kreweras [7] gave the beautiful and surprising result (also see [4]):

Theorem 1. $f\left(n_{1}, n_{2}, \cdots, n_{p}\right)=n(n-1) \cdots(n-p+2) / \prod_{k \geq 1} p_{k}$ !

Namely, $f\left(n_{1}, n_{2}, \cdots, n_{p}\right)$ depends on $n_{1}, n_{2}, \cdots, n_{p}$ only through $p_{k}$. An immediate consequence is that if the $n_{i}$ 's are distinct, then $f\left(n_{1}, n_{2}, \cdots, n_{p}\right)=$ $n(n-1) \cdots(n-p+2)$, independently of $n_{1}, n_{2}, \cdots, n_{p}$. Kreweras' proof resorts to a combinatorial equality derived in another paper [8]. In this paper we give a direct, entirely bijective, proof starting from the same initial idea as Kreweras' proof.

\section{A simple PRoOf of the theOREM}

We give a vector representation of a noncrossing partition.

Received by the editors November 6, 1996.

1991 Mathematics Subject Classification. Primary 05A18.

Liaw, Yeh, and Chang were supported in part by the National Science Council under grant NSC86-2115-M009-002.

(C) 1998 American Mathematical Society 
Lemma 2. Suppose the parts are distinguishable. Then there is a 1-1 onto mapping between the set $\mathcal{N}$ of noncrossing partitions of $[n]$ into $p$ parts with given sizes $n_{1}, n_{2}, \cdots, n_{p}$ and the set $\mathcal{V}$ of vectors $\left(k_{1}, k_{2}, \cdots, k_{p-1}\right)$ where $1 \leq k_{i} \leq n$ and the $k_{i}$ 's are distinct for $1 \leq i \leq p-1$.

Proof. Suppose $\pi \in \mathcal{N}$ and $s$ is the maximum element of $\pi_{p}$. For $1 \leq i \leq p-1$, choose $k_{i}$ as the first element of $\pi_{i}$ when we traverse the cycle from $s$ clockwise. (Actually, any $s \in \pi_{p}$ would give the same $k_{i}$ 's.) Let $g(\pi)=\left(k_{1}, k_{2}, \cdots, k_{p-1}\right)$. Then $g$ is clearly a mapping from $\mathcal{N}$ to $\mathcal{V}$.

Conversely, suppose $\left(k_{1}, k_{2}, \cdots, k_{p-1}\right) \in \mathcal{V}$. We shall construct a unique noncrossing partition $\pi$ as follows. Initially, all elements $1,2, \cdots, n$ in the cycle are unmarked. We perform the following two steps.

Step 1. Find the first unmarked $k_{i}$ such that the number of unmarked elements from $k_{i}$ to the next unmarked $k_{i^{\prime}}$ (including $k_{i}$ but not $k_{i^{\prime}}$ ) is at least $n_{i}$. Choose the first $n_{i}$ such elements in clockwise order to form $\pi_{i}$, and mark them off.

Step 2. Go back to Step 1 until all $k_{i}$ are marked. The remaining elements form $\pi_{p}$.

Note that in Step 1, such a $k_{i}$ always exists since the number of unmarked elements is equal to $n_{p}$ plus the sum of those $n_{j}$ 's for which $k_{j}$ is unmarked. Note also that the construction ensures that the partition $\pi$ is noncrossing. Hence $h\left(k_{1}, k_{2}, \cdots, k_{p-1}\right)=\pi$ is a mapping from $\mathcal{V}$ to $\mathcal{N}$.

For any $\pi^{\prime} \in \mathcal{N}$, let $\left(k_{1}, k_{2}, \cdots, k_{p-1}\right)=g\left(\pi^{\prime}\right)$. Construct $\pi$ from $\left(k_{1}, \cdots, k_{p-1}\right)$ according to Steps 1 and 2 . We prove $\pi=\pi^{\prime}$. Suppose in the construction of $\pi$, the first iteration of Step 1 identifies $k_{i}$. Then $\pi_{i}=\left\{k_{i}, k_{i}+1, \cdots, k_{i}+n_{i}-1\right\}$. Note that $\pi_{i}^{\prime}$ also starts with $k_{i}$. Furthermore, for $j \neq i, k_{j}$ does not lie between $k_{i}$ and $k_{i}+n_{i}-1$ or Step 1 would not identify $k_{i}$. Thus no element of $\pi_{j}^{\prime}$ for all $j \neq i$ and $j \neq p$ can lie between $k_{i}$ and $k_{i}+n_{i}-1$. Finally, no element of $\pi_{p}^{\prime}$ can lie between $k_{i}$ and $k_{i}+n_{i}-1$, for otherwise all elements of $\pi_{p}^{\prime}$ would lie between $k_{i}$ and $k_{i}+n_{i}-1$ and, starting from $s$, the first element of $\pi_{i}^{\prime}$ would not be $k_{i}$. Therefore $\pi_{i}^{\prime}=\pi_{i}$. By deleting $\pi_{i}^{\prime}$ and $\pi_{i}$ from $\pi^{\prime}$ and $\pi$ respectively, a similar argument holds for the part chosen in the second iteration of Step 1, and so on for the third, fourth, ..., iteration.

On the other hand, for $\left(k_{1}^{\prime}, k_{2}^{\prime}, \cdots, k_{p-1}^{\prime}\right) \in \mathcal{V}$, let $\pi=h\left(k_{1}^{\prime}, k_{2}^{\prime}, \cdots, k_{p-1}^{\prime}\right)$. We prove $g(\pi)=\left(k_{1}^{\prime}, k_{2}^{\prime}, \cdots, k_{p-1}^{\prime}\right)$. Consider the step in the construction of $\pi$ when $\pi_{i}$ is chosen to be marked. There is no unmarked element lying between the first and the last elements of $\pi_{i}$ in the clockwise order of the cycle. Hence when we traverse the cycle from any unmarked element, in particular, from $s$, the first element of $\pi_{i}$ we encounter must be $k_{i}^{\prime}$. This shows that $k_{i}^{\prime}$ is the same $k_{i}$ in the definition of $g(\pi)$.

Therefore $h$ is the inverse of $g$. Thus $g$ and $h$ are 1-1 and onto.

Proof of Theorem 1. First suppose that the parts are distinguishable. Then, by Lemma $2,|\mathcal{N}|=|\mathcal{V}|=n(n-1) \cdots(n-p+2)$. However, when $n_{i}=n_{j}$, then interchanging the elements of $\pi_{i}$ and $\pi_{j}$ (including $\pi_{p}$ ) does not lead to a different partition, since parts can be identified only through their sizes. Thus we must divide by $\prod_{k \geq 1} p_{k}$ !. 


\section{ACKNOWLEDGMENTS}

The authors wish to thank the referee for many constructive suggestions on the original version of the paper.

\section{REFERENCES}

[1] H. W. Becker, Planar rhyme schemes, Bull. Amer. Math. Soc. 58 (1952) 39.

[2] J. Bonin, L. Shapiro, and R. Simion, Some $q$-analogues of the Schröder numbers arising from combinatorics on lattice paths, J. Stat. Planning \& Inference 34 (1993) 35-55. MR 94e:05029

[3] A. Denise and R. Simion, Two combinatorial statistics on Dyck paths, Disc. Math. 135 (1995) 155-176. MR 96e:05010

[4] N. Dershowitz and S. Zaks, Ordered trees and non-crossing partitions, Disc. Math. 62 (1986) 215-218. MR 88c:05008

[5] P. H. Edelman, Chain enumeration and non-crossing partitions, Disc. Math. 31 (1980) 171180. MR 81i:01018

[6] P. H. Edelman and R. Simion, Chains in the lattice of noncrossing partitions, Disc. Math. 126 (1994) 107-119. MR 95f:05012

[7] G. Kreweras, Sur les partitions non croisées d'un cycle, Disc. Math. 1 (1972) 333-350. MR 46: 8852

[8] G. Kreweras, Une famille d'identités mettant en jeu toutes les partitions d'un ensemble fini de variables en un nombre donné de classes, C. R. Acad. Sci. Paris 270 (1970) 1140-1143. MR 41:3291

[9] A. Nica and R. Speicher, A "Fourier transform" for multiplicative functions on non-crossing partitions, J. Algeb. Comb., 6 (1997), 141-160. CMP 97:09

[10] Y. Poupard, Étude et dénombrement parallèles des partitions non croisées d'un cycle et des découpages d'un polygone convexe, Disc. Math. 2 (1972) 279-288. MR 46:3369

[11] R. Simion, Combinatorial statistics on non-crossing partitions, J. Comb. Theory, Series A 66 (1994) 270-301. MR 95e:05009

[12] R. Simion and D. Ullman, On the structure of the lattice on non-crossing partitions, Disc. Math. 98 (1991) 193-206. MR 92j:06003

[13] R. Speicher, Multiplicative functions on the lattice of noncrossing partitions and free convolution, Math. Ann. 298 (1994) 611-628. MR 95h:05012

[14] R. P. Stanley, Parking functions and noncrossing partitions, Elect. J. Comb. 4 (1997), no. 2, res. paper 20, approx. 14 pp. (electronic). CMP 97:11

Department of Applied Mathematics, National Chiao Tung University, Hsinchu 30050, TAIWAN

E-mail address: gjchang@math.nctu.edu.tw 